\title{
Forms of Solutions for Some Two-Dimensional Systems of Rational Partial Recursion Equations
}

\author{
Tarek F. Ibrahim ${ }^{1,2}$ and A. Q. Khan (D) $^{3}$ \\ ${ }^{1}$ Department of Mathematics, Faculty of Science, Mansoura University, Mansoura, Egypt \\ ${ }^{2}$ Department of Mathematics, Faculty of Sciences and Arts, King Khalid University, Abha, Saudi Arabia \\ ${ }^{3}$ Department of Mathematics, University of Azad Jammu and Kashmir, Muzaffarabad 13100, Pakistan
}

Correspondence should be addressed to A. Q. Khan; abdulqadeerkhan1@gmail.com

Received 16 March 2021; Revised 23 March 2021; Accepted 24 March 2021; Published 28 April 2021

Academic Editor: Praveen Agarwal

Copyright (C) 2021 Tarek F. Ibrahim and A. Q. Khan. This is an open access article distributed under the Creative Commons Attribution License, which permits unrestricted use, distribution, and reproduction in any medium, provided the original work is properly cited.

\begin{abstract}
In this paper, we offer the closed-form expressions of systems of second-order partial difference equations. We will utilize an alternative approach to verify the results by (odd-even) dual mathematical induction. We research and enforce the specific solutions of partial difference formulas and ordinary difference formulas as a straight effect.
\end{abstract}

\section{Introduction}

Partial difference and differential equations are common in mathematically oriented scientific areas, such as physics and engineering. They are fundamental in the modern-day scientific understanding of mechanical engineering analysis, sound, heat, diffusion, electrodynamics, electrostatics, fluid dynamics, elasticity, general relativity, and also quantum mechanics (see for instance [1-3]). We discover both ordinary and partial difference formulas in probability, characteristics, and even other mathematical physics locations. Certainly, Laplace, as well as Lagrange, took into consideration the solution of partial difference formulas in their research of characteristics as well as probability. An instance of a partial difference formula is the following famous relationship:

$$
C_{s}^{(t)}=C_{s-1}^{(t-1)}+C_{s}^{(t-1)}, \quad 1 \leq s<t .
$$

Some authors research solutions for partial difference formulas.

For instance, Heins [4] considered the solution of

$$
Q_{t+1, s}+Q_{t-1, s}=2 Q_{t, s+1} \text {, }
$$

under some conditions.
In [5], Carlitz researched a solution of

$$
Q_{t, s}-Q_{t, s-1}-Q_{t-1, s}-Q_{t, s-2}+3 Q_{t-1, s-1}-Q_{t-2, s}=0 .
$$

For more outcomes on partial difference equations, we refer the reader to [3, 6-35].

In our work, we have actually researched the solutions complying with systems of partial difference formulas:

$$
\begin{gathered}
\alpha Q_{t, s}+\beta Q_{t, s} Q_{t-2, s-2} R_{t-1, s-1}-Q_{t-2, s-2}=0, \\
\gamma R_{t, s}+\delta R_{t, s} R_{t-2, s-2} Q_{t-1, s-1}-R_{t-2, s-2}=0,
\end{gathered}
$$

where $t, s \in \mathbb{N}_{0}, \mathbb{N}_{0}=\mathbb{N} \cup\{0\}, \alpha, \beta, \gamma, \delta \in\{1,-1\}$, and initial $Q_{t, 0}, Q_{t,-1}, Q_{0, s}, Q_{-1, s}, \quad R_{t, 0}, R_{t,-1}, R_{0, s}$, and $R_{-1, s}$ are real numbers.

As a straight effect, we can derive the solutions of a family of partial difference equations:

$$
\alpha Q_{t, s}+\beta Q_{t, s} Q_{t-2, s-2} Q_{t-1, s-1}-Q_{t-2, s-2}=0,
$$

where $t, s \in \mathbb{N}_{0}, \mathbb{N}_{0}=\mathbb{N} \cup\{0\}, \alpha, \beta \in\{1,-1\}$, and initials $Q_{t, 0}, Q_{t,-1}, Q_{0, s}$, and $Q_{-1, s}$ are real numbers.

In addition, we can acquire the precise solution adhering to systems of ordinary difference formulas: 


$$
\begin{gathered}
\alpha Q_{t}+\beta Q_{t} Q_{t-2} R_{t-1}-Q_{t-2}=0, \\
\gamma R_{t}+\delta R_{t} R_{t-2} Q_{t-1}-R_{t-2}=0,
\end{gathered}
$$

where $t \in \mathbb{N}_{0}, \mathbb{N}_{0}=\mathbb{N} \cup\{0\}, \alpha, \beta, \gamma, \delta \in\{1,-1\}$, and initials $Q_{0}, Q_{-1}, R_{0}$, and $R_{-1}$ are real numbers.

\section{Solution Forms}

We give solutions of systems (4) and (5) for values of $\alpha, \beta, \gamma, \delta \in\{1,-1\}$. This system can be rewritten as

$$
\begin{aligned}
Q_{t, s} & =\frac{Q_{t-2, s-2}}{\alpha+\beta Q_{t-2, s-2} R_{t-1, s-1}}, \\
R_{t, s} & =\frac{R_{t-2, s-2}}{\gamma+\delta R_{t-2, s-2} Q_{t-1, s-1}} .
\end{aligned}
$$

2.1. When $(\alpha, \beta)=(-1,1)$ and $(\gamma, \delta)=(-1,-1)$.

$$
\begin{aligned}
Q_{t, s} & =\frac{Q_{t-2, s-2}}{-1+Q_{t-2, s-2} R_{t-1, s-1}}, \\
R_{t, s} & =\frac{R_{t-2, s-2}}{-1-R_{t-2, s-2} Q_{t-1, s-1}} .
\end{aligned}
$$

Theorem 1. Let $\left\{Q_{t, s}, R_{t, s}\right\}_{t, s=-k}^{\infty}$ be a solution of (9) with

$$
Q_{t, 0}, Q_{t,-1}, Q_{0, s}, Q_{-1, s}, R_{t, 0}, R_{t,-1}, R_{0, s}, R_{-1, s},
$$

where $t, s \in \mathbb{N}_{0}$ and $\mathbb{N}_{0}=\mathbb{N} \cup\{0\}$. Suppose $Q_{-1, s-2} R_{0, s-1} \neq 1$, $Q_{t-2,-1} R_{t-1,0} \neq 1, R_{-1, s-2} Q_{0, s-1} \neq-1$, and $R_{t-2,-1} Q_{t-1,0} \neq-1$. Then, the solutions of (9), for $t, s \geq 1$ and $t \geq s$, are

$$
\begin{aligned}
& Q_{t, s}= \begin{cases}(-1)^{(s / 2)} Q_{t-s, 0} \prod_{k=0}^{(s-2) / 2} \frac{1+(2 k+1) Q_{t-s, 0} R_{t-s-1,-1}}{1+(2 k+2) Q_{t-s, 0} R_{t-s-1,-1}}, & s \text { even, } \\
(-1)^{(t+1) / 2} Q_{t-s-1,-1} \prod_{k=0}^{(s-1) / 2} \frac{-1+(2 k) Q_{t-s-1,-1} R_{t-s, 0}}{-1+(2 k+1) Q_{t-s-1,-1} R_{t-s, 0}}, & s \text { odd },\end{cases} \\
& R_{t, s}= \begin{cases}(-1)^{(s / 2)} R_{t-s, 0} \prod_{k=0}^{(s-2) / 2} \frac{-1+(2 k+1) R_{t-s, 0} Q_{t-s-1,-1}}{-1+(2 k+2) R_{t-s, 0} Q_{t-s-1,-1}} & s \text { even, } \\
(-1)^{(t+1) / 2} R_{t-s-1,-1} \prod_{k=0}^{(s-1) / 2} \frac{1+(2 k) R_{t-s-1,-1} Q_{t-s, 0}}{1+(2 k+1) R_{t-s-1,-1} Q_{t-s, 0}}, & s \text { odd }\end{cases} \\
& Q_{s, n}= \begin{cases}(-1)^{(t+1) / 2} Q_{-1, t-s-1} \prod_{k=0}^{(s-1) / 2} \frac{-1+(2 k) Q_{-1, t-s-1} R_{0, t-s}}{-1+(2 k+1) Q_{-1, t-s-1} R_{0, t-s}}, & s \text { odd, } \\
(-1)^{(s / 2)} Q_{0, t-s} \prod_{k=0}^{(s-2) / 2} \frac{1+(2 k+1) Q_{0, t-s} R_{-1, t-s-1}}{1+(2 k+2) Q_{0, t-s} R_{-1, t-s-1}}, & s \text { even, }\end{cases} \\
& R_{s, n}= \begin{cases}(-1)^{(t+1) / 2} R_{-1, t-s-1} \prod_{k=0}^{(s-1) / 2} \frac{1+(2 k) R_{-1, t-s-1} Q_{0, t-s}}{1+(2 k+1) R_{-1, t-s-1} Q_{0, t-s}}, & s \text { odd, } \\
(-1)^{(s / 2)} R_{0, t-s} \prod_{k=0}^{(s-2) / 2} \frac{-1+(2 k+1) R_{0, t-s} Q_{-1, t-s-1}}{-1+(2 k+2) R_{0, t-s} Q_{-1, t-s-1}}, & s \text { even. }\end{cases}
\end{aligned}
$$

Proof. From system (9), we see the following:

$$
\begin{gathered}
Q_{1,1}=\frac{Q_{-1,-1}}{-1+Q_{-1,-1} R_{0,0}}=-Q_{-1,-1} \prod_{k=0}^{(1-1) / 2} \frac{-1+(2 k) Q_{-1,-1} R_{0,0}}{-1+(2 k+1) Q_{-1,-1} R_{0,0}}, \\
R_{1,1}=\frac{R_{-1,-1}}{-1-R_{-1,-1} Q_{0,0}}=-R_{-1,-1} \prod_{k=0}^{(1-1) / 2} \frac{1+(2 k) R_{-1,-1} Q_{0,0}}{1+(2 k+1) R_{-1,-1} Q_{0,0}} .
\end{gathered}
$$


Now, we prove that $(11)-(14)$ hold for $(t, s)=(2,2)$ :

$$
\begin{aligned}
Q_{2,2} & =\frac{Q_{0,0}}{-1+Q_{0,0} R_{1,1}}=\frac{Q_{0,0}}{-1+Q_{0,0}\left(R_{-1,-1} /\left(-1-R_{-1,-1} Q_{0,0}\right)\right)}=-Q_{0,0}\left(\frac{1+Q_{0,0} R_{-1,-1}}{1+2 Q_{0,0} R_{-1,-1}}\right) \\
& =-Q_{0,0} \prod_{k=0}^{(2-2) / 2} \frac{1+(2 k+1) Q_{0,0} R_{-1,-1}}{1+(2 k+2) Q_{0,0} R_{-1,-1}}, \\
R_{2,2} & =\frac{R_{0,0}}{-1-R_{0,0} Q_{1,1}}=\frac{-1-R_{0,0}\left(Q_{-1,-1} /\left(-1-Q_{-1,-1} R_{0,0}\right)\right)}{-1}=-R_{0,0}\left(\frac{1-R_{0,0} Q_{-1,-1}}{1-2 R_{0,0} Q_{-1,-1}}\right) \\
& =-R_{0,0} \prod_{k=0}^{(2-2) / 2} \frac{-1+(2 k+1) R_{0,0} Q_{-1,-1}}{-1+(2 k+2) R_{0,0} Q_{-1,-1}}
\end{aligned}
$$

Moreover, we prove that (11)-(14) hold for $(t, s)=(1,2)$ and $(t, s)=(2,1)$ :

$$
\begin{aligned}
& Q_{1,2}=\frac{Q_{-1,0}}{-1+Q_{-1,0} R_{0,1}}=-Q_{-1,0} \prod_{k=0}^{(1-1) / 2} \frac{-1+(2 k) Q_{-1,0} R_{0,1}}{-1+(2 k+1) Q_{-1,0} R_{0,1}}, \\
& R_{1,2}=\frac{R_{-1,0}}{-1-R_{-1,0} Q_{0,1}}=-R_{-1,0} \prod_{k=0}^{(1-1) / 2} \frac{1+(2 k) R_{-1,0} Q_{0,1}}{1+(2 k+1) R_{-1,0} Q_{0,1}}, \\
& Q_{2,1}=\frac{Q_{0,-1}}{-1+Q_{0,-1} R_{1,0}}=-Q_{0,-1} \prod_{k=0}^{(1-1) / 2} \frac{-1+(2 k) Q_{0,-1} R_{1,0}}{-1+(2 k+1) Q_{0,-1} R_{1,0}}, \\
& R_{2,1}=\frac{R_{0,-1}}{-1-R_{0,-1} Q_{1,0}}=-R_{0,-1} \prod_{k=0}^{(1-1) / 2} \frac{1+(2 k) R_{0,-1} Q_{1,0}}{1+(2 k+1) R_{0,-1} Q_{1,0}} .
\end{aligned}
$$

Now, suppose that (11)-(14) hold for $s=1$ and $s=2$ with $t \in \mathbb{N}$. So,

$$
\begin{aligned}
Q_{t, 1} & =-Q_{t-2,-1} \prod_{k=0}^{0} \frac{-1+(2 k) Q_{t-2,-1} R_{t-1,0}}{-1+(2 k+1) Q_{t-2,-1} R_{t-1,0}} \\
& =\frac{-Q_{t-2,-1}}{1-Q_{t-2,-1} R_{t-1,0}}, \\
R_{t, 1} & =\frac{-R_{t-2,-1}}{1+R_{t-2,-1} Q_{t-1,0}}, \\
Q_{t, 2} & =-Q_{t-2,0}\left(\frac{1+Q_{t-2,0} R_{t-3,-1}}{1+2 Q_{t-2,0} R_{t-3,-1}}\right), \\
R_{t, 2} & =-R_{t-2,0}\left(\frac{1-R_{t-2,0} Q_{t-3,-1}}{1-2 R_{t-2,0} Q_{t-3,-1}}\right) .
\end{aligned}
$$

$$
\begin{gathered}
Q_{t+2,1}=\frac{Q_{t,-1}}{-1+Q_{t,-1} R_{s+1,0}}=-Q_{t,-1} \prod_{k=0}^{(0 / 2)} \frac{1-(2 k) Q_{t,-1} R_{s+1,0}}{1-(2 k+1) Q_{t,-1} R_{s+1,0}}, \\
R_{t+2,1}=\frac{R_{t,-1}}{-1-R_{t,-1} Q_{s+1,0}}=-R_{t,-1} \prod_{k=0}^{(1-1) / 2} \frac{1+(2 k) R_{t,-1} Q_{s+1,0}}{1+(2 k+1) R_{t,-1} Q_{s+1,0}} .
\end{gathered}
$$

Now, we prove that (11)-(14) hold for $s=2$ with $t+2$ :

$$
\begin{gathered}
Q_{t+2,2}=\frac{Q_{t, 0}}{-1+Q_{t, 0} R_{s+1,1}}=\frac{Q_{t, 0}}{-1+Q_{t, 0}\left(R_{t-1,-1} /\left(-1-R_{t-1,-1} Q_{t, 0}\right)\right)} \\
=\frac{-Q_{t, 0}\left(1+R_{t-1,-1} Q_{t, 0}\right)}{1+2 R_{t-1,-1} Q_{t, 0}}=-Q_{t, 0} \prod_{k=0}^{(2-2) / 2} \frac{1+(2 k+1) Q_{t, 0} R_{t-1,-1}}{1+(2 k+2) Q_{t, 0} R_{t-1,-1}}
\end{gathered}
$$

Now, prove that (11)-(14) hold for $s=1$ with $t+2$ :

Similarly, 
$R_{t+2,2}=\frac{R_{t, 0}}{-1-R_{t, 0} Q_{s+1,1}}=-R_{t, 0} \prod_{k=0}^{(2-2) / 2} \frac{1-(2 k+1) R_{t, 0} Q_{t-1,-1}}{1-(2 k+2) R_{t, 0} Q_{t-1,-1}}$.

From (9), we have

$$
Q_{t, s+2}=\frac{Q_{t-2, s}}{-1+Q_{t-2, s} R_{t-1, s+1}} .
$$

There are four cases:

(1) If $t>s+2$ and $s$ is even,

$$
\begin{aligned}
Q_{t, s+2} & =\frac{Q_{t-2, s}}{-1+Q_{t-2, s} R_{t-1, s+1}} \\
& =\frac{(-1)^{(s / 2)} Q_{t-s-2,0} \prod_{k=0}^{(s-2) / 2}((1+(2 k+1) \Phi) /(1+(2 k+2) \Phi))}{-1+\left((-1)^{(s / 2)} Q_{t-s-2,0} \prod_{k=0}^{(s-2) / 2}((1+(2 k+1) \Phi) /(1+(2 k+2) \Phi))\left((-1)^{(s+2) / 2} R_{t-s-3,-1} \prod_{k=0}^{(s / 2)}((1+(2 k) \Phi) /(1+(2 k+1) \Phi))\right)\right.} \\
& =\frac{(-1)^{(s+2) / 2} Q_{t-s-2,0} \prod_{k=0}^{(s-2) / 2}((1+(2 k+1) \Phi) /(1+(2 k+2) \Phi))}{1+(\Phi / 1+(t+1) \Phi)} \\
& =(-1)^{(s+2) / 2} Q_{t-s-2,0} \prod_{k=0}^{(s / 2)} \frac{1+(2 k+1) \Phi}{1+(2 k+2) \Phi}
\end{aligned}
$$

where $\Phi=Q_{t-s-2,0} R_{t-s-3,-1}$.

(2) If $t>s+2$ and $s$ is odd,

$$
\begin{aligned}
Q_{t, s+2} & =\frac{Q_{t-2, s}}{-1+Q_{t-2, s} R_{t-1, s+1}} \\
& =\frac{(-1)^{(t+1) / 2} Q_{t-s-3,-1} \prod_{k=0}^{(s-1) / 2}((-1+(2 k) \Psi) /(-1+(2 k+1) \Psi))}{-1+\left((-1)^{(t+1) / 2} Q_{t-s-3,-1} \prod_{k=0}^{(s-1) / 2}((-1+(2 k) \Psi) /(-1+(2 k+1) \Psi))\left((-1)^{(t+1) / 2} R_{t-s-2,0} \prod_{k=0}^{(s-1) / 2}((-1+(2 k+1) \Psi) /(-1+(2 k+2) \Psi))\right)\right.} \\
& =\frac{(-1)^{(t+1) / 2} Q_{t-s-3,-1} \prod_{k=0}^{(s-1) / 2}((-1+(2 k) \Psi) /(-1+(2 k+1) \Psi))}{-1-(\Psi /-1+(t+1) \Psi)} \\
& =(-1)^{(s+3) / 2} Q_{t-s-3,-1} \prod_{k=0}^{(t+1) / 2} \frac{-1+(2 k) \Psi}{-1+(2 k+1) \Psi}
\end{aligned}
$$

where $\Psi=Q_{t-s-3,-1} R_{t-s-2,0}$.

(3) This is as in part (1)

(4) This is as in part (2)

Proposition 1. Properties for system (9) are as follows:

(1) If $s$ is even and $Q_{t-s, 0}=0$, then $Q_{t, s}=0$

(2) If $s$ is odd and $Q_{t-s, 0}=0$, then $R_{t, s}=(-1)^{(t+1) / 2}$ $R_{t-s-1,-1}$

(3) If $s$ is even and $R_{t-s, 0}=0$, then $R_{t, s}=0$

(4) If $s$ is odd and $R_{t-s, 0}=0$, then $Q_{t, s}=(-1)^{(t+1) / 2}$ $Q_{t-s-1,-1}$

(5) If $s$ is even and $Q_{t-s-1,-1}=0$, then $R_{t, s}=(-1)^{(s / 2)} R_{t-s, 0}$

(6) If $s$ is odd and $Q_{t-s-1,-1}=0$, then $Q_{t, s}=0$

(7) If $s$ is even and $R_{t-s-1,-1}=0$, then $Q_{t, s}=(-1)^{(s / 2)}$ $Q_{t-s, 0}$
(8) If $s$ is odd and $R_{t-s-1,-1}=0$, then $R_{t, s}=0$

Proposition 2. We have the following properties for system (9):

(1) If $s$ is even and $Q_{0, t-s}=0$, then $Q_{s, t}=0$

(2) If $s$ is odd and $Q_{0, t-s}=0$, then $R_{s, t}=(-1)^{(t+1) / 2}$ $R_{-1, t-s-1}$

(3) If $s$ is even and $R_{0, t-s}=0$, then $R_{s, t}=0$

(4) If $s$ is odd and $R_{0, t-s}=0$, then $Q_{s, t}=(-1)^{(t+1) / 2}$ $Q_{-1, t-s-1}$

(5) If $s$ is even and $Q_{-1, t-s-1}=0$, then $R_{s, t}=(-1)^{(s / 2)} R_{0, t-s}$

(6) If $s$ is odd and $Q_{-1, t-s-1}=0$, then $Q_{s, t}=0$

(7) If $s$ is even and $R_{-1, t-s-1}=0$, then $Q_{s, t}=(-1)^{(s / 2)}$ $Q_{0, t-s}$

(8) If $s$ is odd and $R_{-1, t-s-1}=0$, then $R_{s, t}=0$ 
Remark 1. As a special case of (9), we have

$$
\begin{aligned}
Q_{t} & =\frac{Q_{t-2}}{-1+Q_{t-2} R_{t-1}}, \\
R_{t} & =\frac{R_{t-2}}{-1-R_{t-2} Q_{t-1}} .
\end{aligned}
$$

Corollary 1. Let $\left\{Q_{t}, R_{t}\right\}_{t=-k}^{\infty}$ be a solution of (25) with initials $Q_{0}, Q_{-1}, R_{0}$, and $R_{-1}$. Suppose $Q_{-1} R_{0} \neq 1$ and $R_{-1} Q_{0} \neq-1$. Then,

$$
\begin{aligned}
& Q_{t}= \begin{cases}(-1)^{(t / 2)} Q_{0} \prod_{k=0}^{(t-2) / 2} \frac{1+(2 k+1) Q_{0} R_{-1}}{1+(2 k+2) Q_{0} R_{-1}}, & t \text { even, } \\
(-1)^{(t+1) / 2} Q_{-1} \prod_{k=0}^{(t-1) / 2} \frac{-1+(2 k) Q_{-1} R_{0}}{-1+(2 k+1) Q_{-1} R_{0}} & t \text { odd, }\end{cases} \\
& R_{t}= \begin{cases}(-1)^{(t / 2)} R_{0} \prod_{k=0}^{(t-2) / 2} \frac{-1+(2 k+1) R_{0} Q_{-1}}{-1+(2 k+2) R_{0} Q_{-1}}, & t \text { even, } \\
(-1)^{(t+1) / 2} R_{-1} \prod_{k=0}^{(t-1) / 2} \frac{1+(2 k) R_{-1} Q_{0}}{1+(2 k+1) R_{-1} Q_{0}} & t \text { odd. }\end{cases}
\end{aligned}
$$

2.2. When $(\alpha, \beta)=(\gamma, \delta)=(-1,-1)$.

$$
\begin{aligned}
Q_{t, s} & =\frac{Q_{t-2, s-2}}{-1-Q_{t-2, s-2} R_{t-1, s-1}}, \\
R_{t, s} & =\frac{R_{t-2, s-2}}{-1-R_{t-2, s-2} Q_{t-1, s-1}} .
\end{aligned}
$$

Theorem 2. Let $\left\{Q_{t, s}, R_{t, s}\right\}_{t, s=-k}^{\infty}$ be a solution of (27) with $Q_{t, 0}, Q_{t,-1}, Q_{0, s}, Q_{-1, s}, R_{t, 0}, R_{t,-1}, R_{0, s}$, and $R_{-1, s}$ where $t, s \in \mathbb{N}_{0}$ and $\mathbb{N}_{0}=\mathbb{N} \cup\{0\}$. Suppose $Q_{-1, s-2} R_{0, s-1} \neq-1, Q_{t-2,-1}$ $R_{t-1,0} \neq-1, \quad R_{-1, s-2} Q_{0, s-1} \neq-1, \quad$ and $R_{t-2,-1} Q_{t-1,0} \neq-1$. Then,

$$
\begin{aligned}
& Q_{t, s}= \begin{cases}\frac{(-1)^{(t+1) / 2} Q_{t-s-1,-1}}{\left(1+Q_{t-s-1,-1} R_{t-s, 0}\right)^{(t+1) / 2}}, & s \text { odd; } \\
(-1)^{(s / 2)} Q_{t-s, 0}\left(1+Q_{t-s, 0} R_{t-s-1,-1}\right)^{(s / 2)}, & s \text { even, }\end{cases} \\
& R_{t, s}= \begin{cases}\frac{(-1)^{(t+1) / 2} R_{t-s-1,-1}}{\left(1+R_{t-s-1,-1} Q_{t-s, 0}\right)^{(t+1) / 2}}, & s \text { odd } \\
(-1)^{(s / 2)} R_{t-s, 0}\left(1+R_{t-s, 0} Q_{t-s-1,-1}\right)^{(s / 2)}, & s \text { even, }\end{cases} \\
& Q_{s, t}= \begin{cases}\frac{(-1)^{(t+1) / 2} Q_{-1, t-s-1}}{\left(1+Q_{-1, t-s-1} R_{0, t-s}\right)^{(t+1) / 2}}, & s \text { odd; } \\
(-1)^{(s / 2)} Q_{0, t-s}\left(1+Q_{0, t-s} R_{-1, t-s-1}\right)^{(s / 2)}, & s \text { even, }\end{cases} \\
& R_{s, t}= \begin{cases}\frac{(-1)^{(t+1) / 2} R_{-1, t-s-1}}{\left(1+R_{-1, t-s-1} Q_{0, t-s}\right)^{(t+1) / 2}}, & s \text { odd } \\
(-1)^{(s / 2)} R_{0, t-s}\left(1+R_{0, t-s} Q_{-1, t-s-1}\right)^{(s / 2)}, & s \text { even. }\end{cases}
\end{aligned}
$$


Proof. The proof is given as in Theorem (1).

Remark 2. If we consider

$$
Q_{t, s}=\frac{Q_{t-2, s-2}}{-1-Q_{t-2, s-2} Q_{t-1, s-1}},
$$

then the solution of equation (29) is as follows.

Corollary 2. Let $\left\{Q_{t, s}\right\}_{t, s=-k}^{\infty}$ be a solution of (29) with $Q_{t, 0}, Q_{t,-1}, Q_{0, s}$, and $Q_{-1, s}$ where $t, s \in \mathbb{N}_{0}$ and $\mathbb{N}_{0}=\mathbb{N} \cup\{0\}$. Suppose $Q_{-1, s-2} Q_{0, s-1} \neq-1$ and $Q_{t-2,-1} Q_{t-1,0} \neq-1$. Then,

$$
\begin{aligned}
& Q_{t, s}= \begin{cases}\frac{(-1)^{(t+1) / 2} Q_{t-s-1,-1}}{\left(1+Q_{t-s-1,-1} Q_{t-s, 0}\right)^{(t+1) / 2}}, & s \text { odd }, \\
(-1)^{(s / 2)} Q_{t-s, 0}\left(1+Q_{t-s, 0} Q_{t-s-1,-1}\right)^{(s / 2)}, & s \text { even, }\end{cases} \\
& Q_{s, t}= \begin{cases}\frac{(-1)^{(t+1) / 2} Q_{-1, t-s-1}}{\left(1+Q_{-1, t-s-1} Q_{0, t-s}\right)^{(t+1) / 2}}, & s \text { odd }, \\
(-1)^{(s / 2)} Q_{0, t-s}\left(1+Q_{0, t-s} Q_{-1, t-s-1}\right)^{(s / 2)}, & s \text { even. }\end{cases}
\end{aligned}
$$

Proposition 3. We have the following:

(1) If $s$ is even and $Q_{t-s, 0}=0$, then $Q_{t, s}=0$

(2) If $s$ is odd and $Q_{t-s, 0}=0$, then $Q_{t, s}=(-1)^{(t+1) / 2}$ $Q_{t-s-1,-1}$

(3) If $s$ is even and $Q_{t-s-1,-1}=0$, then $Q_{t, s}=(-1)^{(s / 2)}$ $Q_{t-s, 0}$

(4) If $s$ is odd and $Q_{t-s-1,-1}=0$, then $Q_{t, s}=0$

(5) If $s$ is even and $Q_{0, t-s}=0$, then $Q_{s, t}=0$

(6) If $s$ is odd and $Q_{0, t-s}=0$, then $Q_{s, t}=(-1)^{(t+1) / 2}$ $Q_{-1, t-s-1}$

(7) If $s$ is even and $Q_{-1, t-s-1}=0$, then $Q_{s, t}=(-1)^{(s / 2)}$ $Q_{0, t-s}$

(8) If $s$ is odd and $Q_{-1, t-s-1}=0$, then $Q_{s, t}=0$

Remark 3. As a special of system (27), we have

$$
\begin{aligned}
Q_{t} & =\frac{Q_{t-2}}{-1-Q_{t-2} R_{t-1}}, \\
R_{t} & =\frac{R_{t-2}}{-1-R_{t-2} Q_{t-1}} .
\end{aligned}
$$

Corollary 3. Let $\left\{Q_{t}, R_{t}\right\}_{t=-k}^{\infty}$ be a solution of (31) with $Q_{0}, Q_{-1}, R_{0}, R_{-1}, Q_{-1} R_{0} \neq-1$, and $R_{-1} Q_{0} \neq-1$. Then,

$$
Q_{t}= \begin{cases}\frac{(-1)^{(t+1) / 2} Q_{-1}}{\left(1+Q_{-1} R_{0}\right)^{(t+1) / 2}} & t \text { odd, } \\ (-1)^{(t / 2)} Q_{0}\left(1+Q_{0} R_{-1}\right)^{(t / 2)}, & t \text { even, }\end{cases}
$$

$$
R_{t}= \begin{cases}\frac{(-1)^{(t+1) / 2} R_{-1}}{\left(1+R_{-1} Q_{0}\right)^{(t+1) / 2},} & t \text { odd } \\ (-1)^{(t / 2)} R_{0}\left(1+R_{0} Q_{-1}\right)^{(t / 2)}, & t \text { even }\end{cases}
$$

Remark 4. As a special case of system (31), we get

$$
Q_{t}=\frac{Q_{t-2}}{-1-Q_{t-2} Q_{t-1}} .
$$

The closed-form solution of (33) is

$$
Q_{t}= \begin{cases}\frac{(-1)^{(t+1) / 2} Q_{-1}}{\left(1+Q_{-1} Q_{0}\right)^{(t+1) / 2}}, & t \text { odd } \\ (-1)^{(t / 2)} Q_{0}\left(1+Q_{0} Q_{-1}\right)^{(t / 2)}, & t \text { even }\end{cases}
$$

where $t \in \mathbb{N}$ and $Q_{-1} Q_{0} \neq-1$.

If $n$ is even (or odd) and $Q_{0}=0$, then $Q_{t}=0\left(Q_{t}=(-1)^{(t+1) / 2} Q_{-1}\right)$.

Also, if $n$ is even (or odd) and $Q_{-1}=0$, then $Q_{t}=(-1)^{(t / 2)} Q_{0}\left(Q_{t}=0\right)$.

2.3. When $(\alpha, \beta)=(1,1)$ and $(\gamma, \delta)=(-1,-1)$.

$$
\begin{aligned}
Q_{t, s} & =\frac{Q_{t-2, s-2}}{1+Q_{t-2, s-2} R_{t-1, s-1}}, \\
R_{t, s} & =\frac{R_{t-2, s-2}}{-1-R_{t-2, s-2} Q_{t-1, s-1}} .
\end{aligned}
$$

Theorem 3. Let $\left\{Q_{t, s}, R_{t, s}\right\}_{t, s=-k}^{\infty}$ be a solution of (35) with $Q_{t, 0}, Q_{t,-1}, Q_{0, s}, Q_{-1, s}, R_{t, 0}, R_{t,-1}, R_{0, s}$, and $R_{-1, s}$ where $t, s \in \mathbb{N}_{0}$ and $\mathbb{N}_{0}=\mathbb{N} \cup\{0\}$. Suppose $Q_{-1, s-2} R_{0, s-1} \neq-1, Q_{t-2,-1}$ $R_{t-1,0} \neq-1, \quad R_{-1, s-2} Q_{0, s-1} \neq-1$, and $R_{t-2,-1} Q_{t-1,0} \neq-1$. Then, 


$$
\begin{aligned}
& Q_{t, s}= \begin{cases}\frac{Q_{t-s-1,-1}\left(1+2 Q_{t-s-1,-1} R_{t-s, 0}\right)^{(s-1) / 4}}{\left(1+Q_{t-s-1,-1} R_{t-s, 0}\right)^{(t+1) / 2}}, & s=4 K+1, \\
(-1)^{(s-2) / 4} Q_{t-s, 0}\left(-1+Q_{t-s, 0} R_{t-s-1,-1}\right)^{(s-2) / 4}\left(1+Q_{t-s, 0} R_{t-s-1,-1}\right)^{(s+2) / 4}, & s=4 K+2, \\
\frac{Q_{t-s-1,-1}\left(1+2 Q_{t-s-1,-1} R_{t-s, 0}\right)^{(t+1) / 4}}{\left(1+Q_{t-s-1,-1} R_{t-s, 0}\right)^{(t+1) / 2}}, & s=4 K+3, \\
(-1)^{(s / 4)} Q_{t-s, 0}\left(-1+Q_{t-s, 0} R_{t-s-1,-1}\right)^{(s / 4)}\left(1+Q_{t-s, 0} R_{t-s-1,-1}\right)^{(s / 4)}, & s=4 K+4,\end{cases} \\
& R_{t, s}= \begin{cases}\frac{(-1)^{(s+3) / 4} R_{t-s-1,-1}}{\left(-1+R_{t-s-1,-1} Q_{t-s, 0}\right)^{(s-1) / 4}\left(1+R_{t-s-1,-1} Q_{t-s, 0}\right)^{(s+3) / 4}}, & s=4 K+1, \\
\frac{(-1)^{(s+2) / 4} R_{t-s, 0}\left(1+R_{t-s, 0} Q_{t-s-1,-1}\right)^{(s / 2)},}{\left(1+2 R_{t-s, 0} Q_{t-s-1,-1}\right)^{(s+2) / 4}}, & s=4 K+2, \\
\frac{(-1)^{(t+1) / 4} R_{t-s-1,-1}}{\left(-1+R_{t-s-1,-1} Q_{t-s, 0}\right)^{(t+1) / 4}\left(1+R_{t-s-1,-1} Q_{t-s, 0}\right)^{(t+1) / 4},} & s=4 K+3, \\
\frac{R_{t-s, 0}\left(1+R_{t-s, 0} Q_{t-s-1,-1}\right)^{(s / 2)},}{\left(1+2 R_{t-s, 0} Q_{t-s-1,-1}\right)^{(s / 4)}} & s=4 K+4,\end{cases} \\
& \int \frac{Q_{-1, t-s-1}\left(1+2 Q_{-1, t-s-1} R_{0, t-s}\right)^{(s-1) / 4}}{\left(1+Q_{-1, t-s-1} R_{0, t-s}\right)^{(t+1) / 2}}, \quad s=4 K+1 \text {, } \\
& Q_{s, t}= \begin{cases}(-1)^{(s-2) / 4} Q_{0, t-s}\left(-1+Q_{0, t-s} R_{-1, t-s-1}\right)^{(s-2) / 4}\left(1+Q_{0, t-s} R_{-1, t-s-1}\right)^{(s+2) / 4}, & s=4 K+2, \\
\frac{Q_{-1, t-s-1}\left(1+2 Q_{-1, t-s-1} R_{0, t-s}\right)^{(t+1) / 4}}{\left(1+Q_{-1, t-s-1} R_{0, t-s}\right)^{(t+1) / 2}}, & s=4 K+3,\end{cases} \\
& (-1)^{(s / 4)} Q_{0, t-s}\left(-1+Q_{0, t-s} R_{-1, t-s-1}\right)^{(s / 4)}\left(1+Q_{0, t-s} R_{-1, t-s-1}\right)^{(s / 4)}, \quad s=4 K+4, \\
& R_{s, n}= \begin{cases}\frac{(-1)^{(s+3) / 4} R_{-1, t-s-1}}{\left(-1+R_{-1, t-s-1} Q_{0, t-s}\right)^{(s-1) / 4}\left(1+R_{-1, t-s-1} Q_{0, t-s}\right)^{(s+3) / 4}}, & s=4 K+1, \\
\frac{(-1)^{(s+2) / 4} R_{0, t-s}\left(1+R_{0, t-s} Q_{-1, t-s-1}\right)^{(s / 2)}}{\left(1+2 R_{0, t-s} Q_{-1, t-s-1}\right)^{(s+2) / 4}}, & s=4 K+2, \\
\frac{(-1)^{(t+1) / 4} R_{-1, t-s-1}}{\left(-1+R_{-1, t-s-1} Q_{0, t-s}\right)^{(t+1) / 4}\left(1+R_{-1, t-s-1} Q_{0, t-s}\right)^{(t+1) / 4},} & s=4 K+3, \\
\frac{R_{0, t-s}\left(1+R_{0, t-s} Q_{-1, t-s-1}\right)^{(s / 2)},}{\left(1+2 R_{0, t-s} Q_{-1, t-s-1}\right)^{(s / 4)}} & s=4 K+4,\end{cases}
\end{aligned}
$$


where $k=0,1,2,3, \ldots$.

Proof. The proof is given by piecewise double mathematical induction.

Proposition 4. We have the following:

(1) If $s$ is even and $Q_{t-s, 0}=0$, then $Q_{t, s}=0$

(2) If $s$ is odd and $Q_{t-s, 0}=0$, then $R_{t, s}= \pm R_{t-s-1,-1}$

(3) If $s$ is even and $R_{t-s, 0}=0$, then $R_{t, s}=0$

(4) If $s$ is odd and $R_{t-s, 0}=0$, then $Q_{t, s}=Q_{t-s-1,-1}$

(5) If $s$ is even and $Q_{t-s-1,-1}=0$, then $R_{t, s}= \pm R_{t-s, 0}$

(6) If $s$ is odd and $Q_{t-s-1,-1}=0$, then $Q_{t, s}=0$

(7) If $s$ is even and $R_{t-s-1,-1}=0$, then $Q_{t, s}=Q_{t-s, 0}$

(8) If $s$ is odd and $R_{t-s-1,-1}=0$, then $R_{t, s}=0$

Proposition 5. We have the following:

(1) If $s$ is even and $Q_{0, t-s}=0$, then $Q_{s, t}=0$

(2) If $s$ is odd and $Q_{0, t-s}=0$, then $R_{s, t}= \pm R_{-1, t-s-1}$
(3) If $s$ is even and $R_{0, t-s}=0$, then $R_{s, t}=0$

(4) If $s$ is odd and $R_{0, t-s}=0$, then $Q_{s, t}=Q_{-1, t-s-1}$

(5) If $s$ is even and $Q_{-1, t-s-1}=0$, then $R_{s, t}= \pm R_{0, t-s}$

(6) If $s$ is odd and $Q_{-1, t-s-1}=0$, then $Q_{s, t}=0$

(7) If $s$ is even and $R_{-1, t-s-1}=0$, then $Q_{s, t}=Q_{0, t-s}$

(8) If $s$ is odd and $R_{-1, t-s-1}=0$, then $R_{s, t}=0$

Remark 5. As a special case,

$$
\begin{aligned}
Q_{t} & =\frac{Q_{t-2}}{1+Q_{t-2} R_{t-1}}, \\
R_{t} & =\frac{R_{t-2}}{-1-R_{t-2} Q_{t-1}} .
\end{aligned}
$$

Corollary 4. Let $\left\{Q_{t}, R_{t}\right\}_{t=-k}^{\infty}$ be a solution of (37) with initials $Q_{0}, Q_{-1}, R_{0}$, and $R_{-1}$. Suppose $Q_{-1} R_{0} \neq-1$ and $R_{-1} Q_{0} \neq-1$. Then,

$$
Q_{t}= \begin{cases}\frac{Q_{-1}\left(1+2 Q_{-1} R_{0}\right)^{(t-1) / 4}}{\left(1+Q_{-1} R_{0}\right)^{(t+1) / 2},} & t=4 K+1, \\ (-1)^{(t-2) / 4} Q_{0}\left(-1+Q_{0} R_{-1}\right)^{(t-2) / 4}\left(1+Q_{0} R_{-1}\right)^{(t+2) / 4}, & t=4 K+2, \\ \frac{Q_{-1}\left(1+2 Q_{-1} R_{0}\right)^{(t+1) / 4}}{\left(1+Q_{-1} R_{0}\right)^{(t+1) / 2},} & t=4 K+3, \\ (-1)^{(t / 4)} Q_{0}\left(-1+Q_{0} R_{-1}\right)^{(t / 4)}\left(1+Q_{0} R_{-1}\right)^{(t / 4)}, & t=4 K+4,\end{cases}
$$

$$
R_{t}= \begin{cases}\frac{(-1)^{(t+3) / 4} R_{-1}}{\left(-1+R_{-1} Q_{0}\right)^{(t-1) / 4}\left(1+R_{-1} Q_{0}\right)^{(t+3) / 4},} & t=4 K+1, \\ \frac{(-1)^{(t+2) / 4} R_{0}\left(1+R_{0} Q_{-1}\right)^{(t / 2)}}{\left(1+2 R_{0} Q_{-1}\right)^{(t+2) / 4}}, & t=4 K+2, \\ \frac{(-1)^{(t+1) / 4} R_{-1}}{\left(-1+R_{-1} Q_{0}\right)^{(t+1) / 4}\left(1+R_{-1} Q_{0}\right)^{(t+1) / 4}}, & t=4 K+3, \\ \frac{R_{0}\left(1+R_{0} Q_{-1}\right)^{(t / 2)}}{\left(1+2 R_{0} Q_{-1}\right)^{(t / 4)}} & t=4 K+4,\end{cases}
$$


where $k=0,1,2,3 \ldots$

\section{Data Availability}

All the data utilized for this study have been included within the article and their sources are cited accordingly.

\section{Conflicts of Interest}

The authors declare that they have no conflicts of interest regarding the publication of this paper.

\section{Acknowledgments}

The Higher Education Commission of Pakistan partially supported A. Q. Khan's research.

\section{References}

[1] P. Agarwal, S. Deniz, S. Jain, A. A. Alderremy, and S. Aly, “A new analysis of a partial differential equation arising in biology and population genetics via semi analytical techniques," Physica A: Statistical Mechanics and Its Applications, vol. 542, p. 122769, 2020.

[2] P. Agarwal, E. Karimov, M. Mamchuev, and M. Ruzhansky, I. Pesenson, "On boundary-value problems for a partial differential equation with Caputo and Bessel operators," in Recent Applications of Harmonic Analysis to Function Spaces, Differential Equations, and Data Science: Novel Methods in Harmonic Analysis, Q. T. L. Gia, A. Mayeli, H. Mhaskar, and D.-X. Zhou, Eds., pp. 707-718, Springer, Cham, Switzerland, 2017.

[3] S. S. Haider, M. Ur Rehman, and T. Abdeljawad, "A transformation method for delta partial difference equations on discrete time scale," Mathematical Problems in Engineering, vol. 2020, Article ID 3902931, 14 pages, 2020.

[4] A. E. Heins, "On the solution of partial difference equations," American Journal of Mathematics, vol. 63, no. 2, pp. 435-442, 1941.

[5] L. Carlitz, "A partial difference equation related to the Fibonacci numbers," Fibonacci Quarterly, vol. 2, no. 3, pp. 185-196, 1964.

[6] M. J. Ablowitz and J. F. Ladik, "On the solution of a class of nonlinear partial difference equations," Studies in Applied Mathematics, vol. 57, no. 1, pp. 1-12, 1977.

[7] F. G. Boese, "Asymptotical stability of partial difference equations with variable coefficients," Journal of Mathematical Analysis and Applications, vol. 276, no. 2, pp. 709-722, 2002.

[8] S. S. Cheng, Partial Difference Equations, Taylor \& Francis, London, UK, 2003.

[9] R. Courant, K. Friedrichs, and H. Lewy, "On the Partial Difference Equations of Mathematical Physics," IBM Journal of Research and Development, vol. 11, no. 2, pp. 215-234, 1967.

[10] E. Elsayed and T. F. Ibrahim, "Periodicity, and solutions for some systems of nonlinear rational difference equations," Hacettepe Journal of Mathematics and Statistics, vol. 44, pp. 1361-1390, 2015.

[11] E. M. Elsayed and T. F. Ibrahim, "Solutions and periodicity of a rational recursive sequences of order five," Bulletin of the Malaysian Mathematical Sciences Society, vol. 38, no. 1, pp. 95-112, 2015.
[12] T. F. Ibrahim, “Generalized partial todd's difference equation in n-dimensional space," Journal of Computational Analysis and Applications, vol. 26, no. 5, pp. 910-926, 2019.

[13] T. F. Ibrahim, "Bifurcation and periodically semicycles for fractional difference equation of fifth order," Journal of Nonlinear Sciences and Applications, vol. 11, no. 3, pp. 375-382, 2018.

[14] T. F. Ibrahim, "Closed form expressions of some systems of nonlinear partial difference equations," Journal of Computational Analysis and Applications, vol. 23, no. 3, pp. 433-445, 2017.

[15] T. F. Ibrahim, "Behavior of two and three-dimensional systems of difference equations in modelling competitive populations," Dynamics of Continuous, Discrete and Impulsive Systems, Series A: Mathematical Analysis, vol. 24, no. 6, pp. 395-418, 2017.

[16] T. F. Ibrahim and M. A. El-Moneam, "Global stability of a higher-order difference equation," Iranian Journal of Science and Technology, Transactions A: Science, vol. 41, no. 1, pp. 51-58, 2017.

[17] T. F. Ibrahim, "Behavior of some higher order nonlinear rational partial difference Equations," Journal of the Egyptian Mathematical Society, vol. 24, no. 4, p. 532, 2016.

[18] T. F. Ibrahim, "Periodicity and global attractivity of difference equation of higher order," Journal of Computational Analysis and Applications, vol. 16, no. 3, pp. 552-564, 2014.

[19] T. F. Ibrahim and N. Touafek, "Max-type system of difference equations with positive two-periodic sequences," Mathematical Methods in the Applied Sciences, vol. 37, no. 16, pp. 2562-2569, 2014.

[20] T. F. Ibrahim, "Boundedness and stability of a rational difference equation with delay," Revue Roumaine des Mathematiques Pures et Appliquees, vol. 57, pp. 215-224, 2012.

[21] T. F. Ibrahim, "Periodicity and analytic solution of a recursive sequence with numerical examples," Journal of Interdisciplinary Mathematics, vol. 12, no. 5, pp. 701-708, 2009.

[22] A. Q. Khan and T. F. Ibrahim, "Stability and bifurcations analysis of discrete dynamical systems," Discrete Dynamics in Nature and Society, vol. 2019, Article ID 8474706, 2 pages, 2019.

[23] A. Q. Khan and S. M. Qureshi, "Dynamical properties of some rational systems of difference equations," Mathematical Methods in the Applied Sciences, vol. 44, no. 5, p. 3485, 2021.

[24] A. Q. Khan, H. M. Arshad, B. A. Younis et al., "Global dynamics of some exponential type systems," Discrete Dynamics in Nature and Society, vol. 202024 pages, 2020.

[25] A. Q. Khan, "Global dynamics of two systems of exponential difference equations by Lyapunov function," Advances in Difference Equations, vol. 297, 2014.

[26] W. Dahmen and C. A. Micchelli, "On the solution of certain systems of partial difference equations and linear dependence of translates of box splines," Transactions of the American Mathematical Society, vol. 292, no. 1, pp. 305-320, 1985.

[27] B. J. Daly, "The stability properties of a coupled pair of nonlinear partial difference equations," Mathematics of Computation, vol. 17, no. 84, pp. 346-360, 1963.

[28] L. Flatto, "Partial differential equations and difference equations," Proceedings of the American Mathematical Society, vol. 16, no. 5, pp. 858-863, 1965.

[29] F. Koehler and C. M. Braden, "An oscillation theorem for solutions of a class of partial difference equations," Proceedings of the American Mathematical Society, vol. 10, no. 5, pp. 762-766, 1959. 
[30] A. C. Newell, "Finite amplitude instabilities of partial difference equations," SIAM Journal on Applied Mathematics, vol. 33, no. 1, pp. 133-160, 1977.

[31] C. Raymond Adams, "Existence theorems for a linear partial difference equation of the intermediate type," Transactions of the American Mathematical Society, vol. 28, no. 1, pp. 119-128, 1926.

[32] I. P. Van den Berg, "On the relation between elementary partial difference equations and partial differential equations," Annals of Pure and Applied Logic, vol. 92, no. 3, pp. 235-265, 1998.

[33] D. Young, "Iterative methods for solving partial difference equations of elliptic type," Transactions of the American Mathematical Society, vol. 76, no. 1, pp. 92-111, 1954.

[34] D. Zeilberger, "Binary operations in the set of solutions of a partial difference equation," Proceedings of the American Mathematical Society, vol. 62, no. 2, pp. 242-244, 1977.

[35] T. F. Ibrahim and Q. Zhang, "Stability of an anti-competitive system of rational difference equations," Archives des Sciences, vol. 66 , no. 5 , pp. $44-58,2013$. 